\title{
Black Hole Remnant in Massive Gravity
}

\author{
B. Eslam Panah,,$^{2,3,4, \dagger}$ S. H. Hendi, ${ }^{3, \ddagger}$ and Y. C. Ong ${ }^{1,5,6,8}$ \\ ${ }^{1}$ Center for Gravitation and Cosmology, College of Physical Science and Technology, \\ Yangzhou University, Yangzhou 225009, China \\ ${ }^{2}$ Research Institute for Astronomy and Astrophysics of Maragha (RIAAM), P.O. Box 55134-441, Maragha, Iran \\ ${ }^{3}$ Physics Department and Biruni Observatory, College of Sciences, Shiraz University, Shiraz 71454, Iran \\ ${ }^{4}$ ICRANet, Piazza della Repubblica 10, I-65122 Pescara, Italy \\ ${ }^{5}$ School of Physics and Astronomy, Shanghai Jiao Tong University, Shanghai 200240, China \\ ${ }^{6}$ Nordita, KTH Royal Institute of Technology \& Stockholm University, \\ Roslagstullsbacken 23, SE-106 91 Stockholm, Sweden
}

\begin{abstract}
The possibility of a nonzero graviton mass has been widely pursued in the literature. In this work we investigate a black hole solution in massive gravity with a degenerate fiducial metric often used in the literature. We find that the end state of Hawking evaporation leads to black hole remnant, which could help to ameliorate the information paradox. We prove that these remnants only exist in anti-de Sitter spacetime. Nevertheless, we speculate on their possible relevance to our Universe as dark matter candidate, in view of the possibility that our Universe could be inherently anti-de Sitter-like, with a transient accelerated expansion phase.
\end{abstract}

\section{INTRODUCTION: A BRIEF ACCOUNT OF MASSIVE GRAVITY}

Black hole remnants are the stable or meta-stable end state of Hawking evaporation, in the sense that Hawking radiation may stop as the mass of the black hole reaches the Planck scale, due to new physics of quantum gravitational nature. They can arise from different theories, or from various quantum gravity inspired phenomenological models. Properties of black hole remnants have been studied in the literature [1, 2]. See [3] for a recent review of the subject. In this paper, our main objective is to find black hole remnants in the theory of massive gravity, without additional gauge fields, which to our knowledge has not been explored before. Given that massive gravity is nowadays a popular candidate for modified theory of gravity (despite its various short comings), this is a topic worth studying - can black hole remnant arise simply by endowing graviton with a mass?

We begin with some background on massive gravity for completeness. Einstein's general relativity can be cast as a theory of massless spin-2 gravitons. Generalizations to massive gravity theories have several motivations, including an attempt to explain the observed accelerated expansion of the Universe. One could also investigate massive gravity as an extension of general relativity, to see if such a theory is consistent, or if general relativity is the unique consistent spin-2 theory of gravitation. Recent observations by LIGO has put a tight bound on graviton's mass [5, 6], but well-known work of Boulware and Deser [7] showed that a generic extension of the Fierz-Pauli (FP) theory [8] to curved backgrounds will give rise to ghost instabilities, now known as the "BD-ghost". A

\footnotetext{
$\dagger$ Electronic address: beslampanah@shirazu.ac.ir

${ }^{\ddagger}$ Electronic address: hendi@shirazu.ac.ir

Electronic address: ycong@yzu.edu.cn
}

special generalization to a nonlinear and stable massive gravity has been introduced by de Rham, Gabadadze and Tolley (dRGT) [9-11], which was subsequently shown to be ghost-free by Hassan and Rosen [12-14]. As we shall see below, dRGT massive gravity comes with two metric tensors, one of which is a fixed spacetime background, as realized by Hassan and Rosen. A natural generalization to having both gravitons being dynamical was then sought by Hassan and Rosen, later known as the bimetric or bigravity theory [15]. However, in this work, we shall focus on the original massive gravity with a fixed background.

In addition to cosmological implications, the nonzero graviton mass allows one to model field theories with momentum dissipation in holography, without the need to employ the more traditional lattice method in the anti-de Sitter bulk [16, 17].

We note that dRGT massive gravity suffers from some problems. Firstly, there is a lack of viable FLRW cosmological solutions [4, 18]. More accurately, massive gravity does not admit FLRW solutions if a flat reference metric is assumed. However such solutions can exist with other choices of the reference metric.

There are also fundamental problems related to the well-posedness of the theory, and "micro-acausality" (arbitrarily small closed causal curve) [19-23] (it is likely that these problems are avoided in the bimetric generalization, given the recent understanding of its complicated causal structure [24]). Nevertheless, there is still merit in further understanding the various aspects of the theory. For example, the effects of nonzero graviton mass on the structure of neutron stars [25] and white dwarfs [26] have been studied recently. The results showed that the maximum mass of these stars can be about three times the solar mass, i.e. more massive than in general relativity.

In this work, we will focus on demonstrating that massive gravity admits black hole remnants. Interestingly, this type of remnants exist in anti-de Sitter spacetime, but not in de Sitter one. We will discuss its possible implications for information paradox of black holes. We also 
speculate on the relevance of these remnants in our actual Universe as possible dark matter candidate; although the Universe is currently undergoing accelerated expansion, it is possible that this is a transient period, and the Universe is actually inherently anti-de Sitter-like. That is to say, current observation does not rule out the possibility that our Universe will be asymptotically anti-de Sitter in the future. The cosmological constant would eventually dominate the evolution of the Universe, slowing its expansion. The current phase of accelerating expansion could be the results of other fields, whose effects might become subdominant compared to the cosmological constant in the far future. A recent study that attempted to address the current tension regarding values of the Hubble constant measured by low $z$ observations and high $z$ Planck measurement from CMB has also proposed such a scenario [27].

\section{BLACK HOLE REMNANTS IN MASSIVE GRAVITY}

The action of dRGT massive gravity can be written as Hilbert-Einstein action with suitable nonlinear interaction terms [10]:

$$
I=\frac{1}{16 \pi} \int \mathrm{d}^{4} x \sqrt{-g}\left(R+m^{2} \mathscr{U}\left(g, \phi^{a}\right)\right),
$$

where $R$ and $\mathscr{U}$ are, respectively, the Ricci scalar and the effective potential of graviton which modifies the gravitational sector with a nonzero graviton mass $m$. Note that despite appearance, dRGT gravity should not be viewed as a "scalar-tensor" theory - the scalar fields are Stückelberg scalars, introduced as a mean to restore the general covariance of the theory [28]. Note that we do not include the cosmological constant a priori in the action, though of course this can be done just as well as in general relativity. The reason for omitting the cosmological constant is in view of the original motivation of the massive gravity theory to explain the accelerating expansion of the Universe without resorting to a cosmological constant.

The Newton constant is dimensionful, but we will set its value as unity for simplicity. This means that graviton mass $m$ has dimension inverse length $(c=1=\hbar)$, but terms like $M / r \equiv G M / c^{2} r$ are dimensionless. This follows the convention of, e.g., [29-31]. The effective potential $\mathscr{U}$ can be written as

$$
\mathscr{U}\left(g, \phi^{a}\right)=\mathscr{U}_{2}+\alpha_{3} \mathscr{U}_{3}+\alpha_{4} \mathscr{U}_{4},
$$

in which $\alpha_{3}$ and $\alpha_{4}$ are two dimensionless free parameters of the theory. The functional form of $\mathscr{U}_{i}$ with respect to the metric $g$ and scalar field $\phi^{\alpha}$ are given by

$$
\begin{aligned}
\mathscr{U}_{2}= & {[\mathscr{K}]^{2}-\left[\mathscr{K}^{2}\right] } \\
\mathscr{U}_{3}= & {[\mathscr{K}]^{3}-3[\mathscr{K}]\left[\mathscr{K}^{2}\right]+2\left[\mathscr{K}^{3}\right] } \\
\mathscr{U}_{4}= & {[\mathscr{K}]^{4}-6\left[\mathscr{K}^{2}\right]\left[\mathscr{K}^{2}+8\left[\mathscr{K}^{3}\right][\mathscr{K}]+3\left[\mathscr{K}^{2}\right]^{2}\right.} \\
& -6\left[\mathscr{K}^{4}\right]
\end{aligned}
$$

in which

$$
\mathscr{K}_{\nu}^{\mu}=\delta_{\nu}^{\mu}-\sqrt{g^{\mu \sigma} f_{a b} \partial_{\sigma} \phi^{a} \partial_{v} \phi^{b}},
$$

where $f_{a b}$ is an appropriate non-dynamical reference metric and the rectangular bracket denotes the traces, namely $[\mathscr{K}]=\mathscr{K}_{\mu}^{\mu}$ and $\left[\mathscr{K}^{n}\right]=\left(\mathscr{K}^{n}\right)_{\mu}^{\mu}$. In addition, $\phi^{a}$ 's are the Stückelberg scalars. In the forthcoming discussions, we shall use the following redefinitions for $\alpha_{3}$ and $\alpha_{4}$, following the convention of [30]

$$
\alpha_{3}=\frac{\alpha-1}{3}, \quad \alpha_{4}=\frac{\beta}{4}+\frac{1-\alpha}{12},
$$

where $\alpha$ and $\beta$ are two arbitrary dimensionless constants.

One finds the following field equation by varying the action with respect to $g_{\mu \nu}$ :

$$
G_{\mu \nu}+m^{2} \chi_{\mu \nu}=0,
$$

where $G_{\mu \nu}$ is the Einstein tensor and $\chi_{\mu \nu}$ takes the form

$$
\begin{aligned}
\chi_{\mu \nu}= & \mathscr{K}_{\mu \nu}-\mathscr{K} g_{\mu \nu}-\alpha\left\{\mathscr{K}_{\mu \nu}^{2}-\mathscr{K} \mathscr{K}_{\mu \nu}+\frac{\mathscr{U}_{2}}{2} g_{\mu \nu}\right\} \\
& +3 \beta^{2}\left\{\mathscr{K}_{\mu \nu}^{3}-\mathscr{K}_{\mu \nu}^{2}+\frac{\mathscr{K}_{\mu \nu}}{2} \mathscr{U}_{2}-\frac{1}{6} g_{\mu \nu} \mathscr{U}_{3}\right\} .
\end{aligned}
$$

We now consider a 4-dimensional static, spherically symmetric spacetime with metric ansatz

$$
\mathrm{d} s^{2}=-g(r) \mathrm{d} t^{2}+\frac{\mathrm{d} r^{2}}{g(r)}+r^{2}\left(\mathrm{~d} \theta^{2}+\sin ^{2} \theta \mathrm{d} \varphi^{2}\right) .
$$

The reference metric essentially plays the role of a Lagrange multiplier to eliminate the BD ghost, and also different choices of the reference metrics give different theories. Here we shall follow the same choice for nondynamical reference metric in the following form [16, 29, 31];

$$
f_{a b}=\operatorname{diag}\left(0,0, c^{2}, c^{2} \sin ^{2} \theta\right),
$$

where $c$ is a positive constant with dimension of length. We should emphasize that the property of massive gravity is such that the choice of reference metric does affect what kind of solutions are allowed, so this black hole solution depends on the choice made above. (For detailed study on black hole solutions in dRGT theory, see [32]) Admittedly, the proof of ghost-freeness of dRGT theory $[13,14]$ assumes that the reference metric is invertible, so for degenerate metric (i.e. its rank is smaller than its dimension) like Eq. (8) one has to analyze the BD ghost separately [16, 17]. It was shown that in [33], the aforementioned non-dynamical reference metric in Eq.(8), does indeed give rise to ghost-freeness. However, since the existence of $\mathrm{BD}$ ghost depends not only on the background but also on the values of free parameters $\alpha$ and $\beta$ (or equivalently $\alpha_{3}$ and $\alpha_{4}$ ), this delicate issue is beyond the scope of the current work. Our aim is less ambitious: taking the theory with the aforementioned reference metric, 
which has been considered numerous times in the literature, what can we say about the existence of black hole remnant?

Indeed, considering the ansatz (7), the reference metric (8), and the field equation (6), we can obtain the following exact solution [30]

$$
g(r)=1-\frac{m_{0}}{r}+\frac{\Lambda r^{2}}{3}+\gamma r+\varepsilon,
$$

where $m_{0}$ is an integration constant related to the mass of the black hole, while $\Lambda, \gamma$ and $\varepsilon$ are, respectively [30],

$$
\begin{aligned}
\Lambda & =3 m^{2}(1+\alpha+\beta), \\
\gamma & =-c m^{2}(1+2 \alpha+3 \beta), \\
\varepsilon & =c^{2} m^{2}(\alpha+3 \beta) .
\end{aligned}
$$

It is notable that by considering the term $3 m^{2}(1+\alpha+$ $\beta$ ) equals to $\Lambda$, one can see that there is a similarity between the obtained black hole solutions in Eq. (9), and the AdS Schwarzschild black holes. So we can consider the term $3 m^{2}(1+\alpha+\beta)$ as the effective cosmological constant. Here we see that the cosmological constant is "emergent" - it comes from the nonzero graviton mass $m$. It follows directly from Eq. (9) that the Schwarzschild solution is recovered for vanishing massive terms $\left(m^{2}=0\right)$. Asymptotically locally anti-de Sitter (AdS)-like and de Sitter (dS)-like solutions are possible (depending on the sign of $(1+\alpha+\beta))$; for nonzero $\gamma$ and $\varepsilon$ the asymptotic geometries are not strictly AdS or dS. The constant term $\varepsilon$ corresponds to global monopole [30].

Now, we briefly discuss the geometrical structure of this solution. For this purpose, we first look for the obvious singularity (if any) by studying two scalar curvatures: Ricci and Kretschmann scalars. Considering the metric (7), with the solution (9), the Ricci scalar is given by

$$
R=-4 \Lambda-\frac{6 \gamma}{r}-\frac{2(1+\gamma)}{r^{2}}
$$

Evidently, we encounter a divergence of the Ricci scalar at the origin $\left(\lim _{r \rightarrow 0} R=\infty\right)$. Also, the Kretschmann scalar $\left(R_{\alpha \beta \gamma \delta} R^{\alpha \beta \gamma \delta}\right)$ is given by a rather lengthy expression:

$$
\begin{aligned}
R_{\alpha \beta \gamma \delta} R^{\alpha \beta \gamma \delta}= & \frac{8 \Lambda^{2}}{3}+\frac{8 \Lambda \gamma}{r}+\frac{8\left(\gamma^{2}+\frac{\Lambda}{3}(1+\varepsilon)\right)}{r^{2}} \\
& +\frac{8 \gamma(1+\varepsilon)}{r^{3}}+\frac{4(1+\varepsilon)^{2}}{r^{4}} \\
& -\frac{8 m_{0}(1+\varepsilon)}{r^{5}}+\frac{12 m_{0}^{2}}{r^{6}}
\end{aligned}
$$

One can show that this scalar has the following behavior

$$
\begin{aligned}
& \lim _{r \longrightarrow 0} R_{\alpha \beta \gamma \delta} R^{\alpha \beta \gamma \delta}=\infty, \\
& \lim _{r \longrightarrow \infty} R_{\alpha \beta \gamma \delta} R^{\alpha \beta \gamma \delta}=\frac{8 \Lambda^{2}}{3},
\end{aligned}
$$

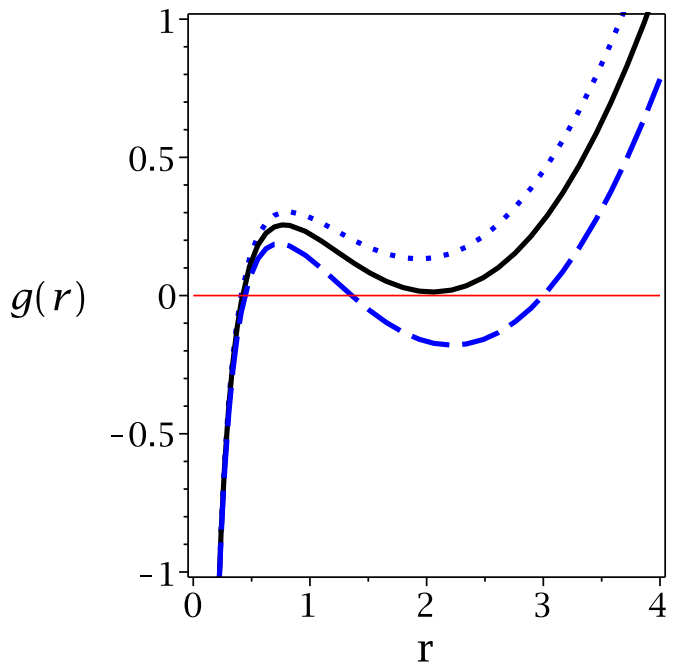

FIG. 1: The plot of $g(r)$ versus $r$, for definiteness here we set $\Lambda=1$, $\varepsilon=1, m_{0}=0.6, \gamma=-1.45$ (dotted line), $\gamma=-1.51$ (continuous line) and $\gamma=-1.60$ (dashed line).

which can confirm that there is a curvature singularity at $r=0$, and also the asymptotic behavior of this solution is $\mathrm{AdS}^{\dagger}$, since the Kretschmann scalar is $\frac{8 \Lambda^{2}}{3}$ at $r \longrightarrow \infty$.

For clarity, we plot the metric function (9) versus $r$ in Fig. (1) for specific choice of the parameter values. As one can see, there is a zero to Eq.(9) which corresponds to the event horizon.

In this work we will show that for black hole remnants to exist, it must be the case that $\Lambda>0$, which, as we have emphasized, from the metric in Eq. (9), actually corresponds to anti-de Sitter case.

The physical mass of the black hole is $M=m_{0} / 2$ [29], which can be obtained from the Hamiltonian method. For $m=0, M$ reduces to the standard ADM mass of an asymptotically flat Schwarzschild black hole. In order to find the event horizon of black hole, we should solve $g(r)=0$. This gives rise to a cubic equation with at most three real roots. For more details see Fig.(1) In fact, the largest real positive root of the function $g(r)$ is the event horizon of the black hole, given by

$$
r_{+}=\frac{\mathscr{A}^{2 / 3}-2 \gamma \mathscr{A}^{1 / 3}-4(1+\varepsilon) \Lambda+4 \gamma^{2}}{2 \Lambda \mathscr{A}^{1 / 3}}
$$

\footnotetext{
$\dagger$ For de-Sitter case, since $r$ is bounded, we cannot take $r \rightarrow \infty$ limit. Note that with the metric Eq. (9), $\Lambda>0$ corresponds to anti-de Sitter instead of de-Sitter. This can be a source of confusion, however we follow the form of Eq. (9) which is widely used in the literature.
} 
where

$$
\begin{aligned}
\mathscr{A}= & 8 \Lambda(1+\varepsilon)\left\{\frac{3 M\left(3 M \Lambda^{2}-2 \gamma^{3}\right)}{(1+\varepsilon)^{2}}+\right. \\
& \left.\frac{\Lambda\left[(1+\varepsilon)^{2}+9 \gamma M\right]}{1+\varepsilon}-\frac{3 \gamma^{2}}{4}\right\}^{\frac{1}{2}}+24 M \Lambda^{2} \\
& +12 \gamma(1+\varepsilon) \Lambda-8 \gamma^{3} .
\end{aligned}
$$

The above root depends on the values of the various parameters. In fact, as the black hole evaporates and the mass decreases, it is possible that the number of real roots changes. Regardless, one identifies the largest root as the event horizon.

The Hawking temperature can be obtained from Eqs. (7), (9) and (15) using the standard method:

$$
T=\left.\frac{1}{4 \pi} g^{\prime}(r)\right|_{r=r_{+}, m_{0}=2 M}=\frac{1+\Lambda r_{+}^{2}+2 \gamma r_{+}+\varepsilon}{4 \pi r_{+}} .
$$

The Bekenstein-Hawking entropy of the black hole can be calculated through the first law of black hole thermodynamics $\mathrm{d} S=\mathrm{d} M / T$, which yields the standard area law upon integration: $S=\pi r_{+}^{2}$. The heat capacity is

$$
C=T\left(\frac{\partial S}{\partial T}\right)=\frac{2 \pi\left(1+\Lambda r_{+}^{2}+2 \gamma r_{+}+\varepsilon\right) r_{+}^{2}}{\Lambda r_{+}^{2}-(1+\varepsilon)} .
$$

As the black hole evaporates, it gradually loses its mass. In general relativity, the temperature of the black hole diverges as $M \rightarrow 0$. This is true even for black holes in antide Sitter spacetime (large enough black holes however, do not evaporate if we impose the usual reflective boundary condition at conformal infinity. See refs. [34, 35], for more details.) Note that, in this work, we consider a model in which the cosmological constant arises from graviton mass $m$, therefore once the parameters $\alpha, \beta$ are fixed to have nonzero $\Lambda, \gamma$ and $\varepsilon$, then general relativity is equivalent to taking $m=0$, i.e. without a cosmological constant. That is to say, the black hole reduces to asymptotically flat Schwarzschild black hole, not Schwarzschild-anti de Sitter black hole.

In various quantum gravity inspired phenomenological models, such as the generalized uncertainty principle, black holes do not evaporate completely but instead become a remnant [36]. Here, we want to show that the black holes in massive gravity do not evaporate completely and we end up with a remnant. For this purpose we solve for the real positive root of the temperature expression set to zero, $T=0$, which is given by following form:

$$
R_{r}=\frac{\sqrt{\gamma^{2}-(1+\varepsilon) \Lambda}-\gamma}{\Lambda} .
$$

The square root actually comes with a \pm sign. However, for definiteness, let us consider the sign in front of the square root to be positive. A black hole remnant also exists if we choose the negative sign.
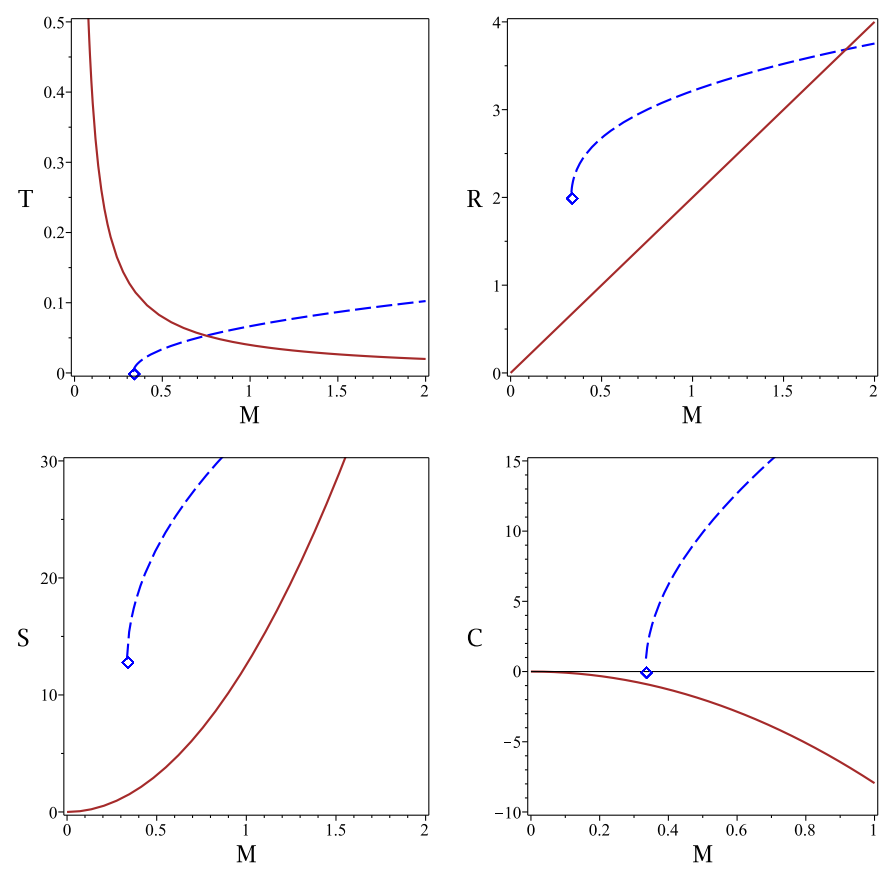

FIG. 2: For this illustrative example we take $\Lambda=1, \varepsilon=1, \gamma=-1.5$. General relativity $(m=0)$ yields asymptotically flat Schwarzschild black hole. Dashed and continuous lines are related to black holes in massive gravity and general relativity, respectively. Up left panel: Temperature $(T)$ versus mass $(M)$. Up right panel: Radius $(R)$ versus mass $(M)$. Down left panel: Entropy $(S)$ versus mass $(M)$. Down right panel: Heat capacity $(C)$ versus mass $(M)$.

By substituting $R_{r}$ in the physical mass $M_{r}\left(r=R_{r}\right)$, one finds that indeed there exists a remnant with mass below which there is no black hole solution:

$$
\begin{aligned}
M_{r}= & \frac{2 \Lambda(1+\varepsilon)+\gamma\left(\sqrt{\gamma^{2}-(1+\varepsilon) \Lambda}-\gamma\right)}{6 \Lambda^{2}} \\
& \times\left(\sqrt{\gamma^{2}-(1+\varepsilon) \Lambda}-\gamma\right) .
\end{aligned}
$$

We remark that in the absence of the cosmological constant the remnant mass of black hole reduces to

$$
M_{r}(\Lambda=0)=-\frac{(1+\varepsilon)^{2}}{8 \gamma} .
$$

In order to have a positive remnant mass of the black hole in the absence of the cosmological constant (Eq. (21)), we always impose $\gamma<0$.

The remnant of entropy is given by

$$
S_{r}=\frac{\pi}{\Lambda^{2}}\left(\sqrt{\gamma^{2}-(1+\varepsilon) \Lambda}-\gamma\right)^{2} .
$$

We present our results in Fig. (2), in which the temperature, radius, entropy and heat capacity of a massive gravity black hole, with parameters chosen to be $\Lambda=1=\varepsilon, \gamma=-1.5$, are depicted and compared against its GR counter-part. We note in particular that the heat capacity and temperature are both zero when the remnant 
mass is reached. Recall that for $\Lambda>0$, our black hole remnant is asymptotically anti-de Sitter.

One might wonder if $\Lambda<0$ solution also exists, which might be more relevant to cosmology? Unfortunately this seems not to be the case. To see this, let us consider Eq. (19) and Eq. (20). We will show that for $\Lambda<0$ (which would correspond to the de Sitter case), it is impossible to obtain remnants for which the mass and the radius are positive simultaneously. With $\Lambda<0$, in Eqs. (19) and (20) can be written as

$$
\begin{aligned}
& R_{r}=\frac{\sqrt{\gamma^{2}+\sigma}-\gamma}{\Lambda} \\
& M_{r}=\frac{\left[-2 \sigma+\gamma\left(\sqrt{\gamma^{2}+\sigma}-\gamma\right)\right]\left(\sqrt{\gamma^{2}+\sigma}-\gamma\right)_{(24)}}{6 \Lambda^{2}}
\end{aligned}
$$

in the above equation $\sigma=-(1+\varepsilon) \Lambda$.

In order to have a real positive remnant radius $\left(R_{r}>0\right)$ for $\Lambda<0$, the following constraints must be satisfied:

$$
\begin{array}{r}
\sqrt{\gamma^{2}+\sigma}-\gamma<0 \\
\gamma^{2}+\sigma>0
\end{array}
$$

\{in which due to Eq. (25), we find the requirement that $\sigma<0$ (or $\varepsilon<-1$ ).

Considering the condition (26), we obtain two possible ranges for $\gamma$ : either $\gamma \geqslant \sqrt{-\sigma}$ or $\gamma \leqslant-\sqrt{-\sigma}$. However, note that Eq. (25) implies that $\gamma>0$, so we must have $\gamma \geqslant \sqrt{-\sigma}$.

\{Now, we focus on the remnant mass of black holes given by Eq. (24). In order to have positive value of remnant mass, the numerator of Eq. (24) has to be positive, which contains two factors:

$$
\begin{aligned}
& \text { (I) }-2 \sigma+\gamma\left(\sqrt{\gamma^{2}+\sigma}-\gamma\right), \\
& \text { (II) } \sqrt{\gamma^{2}+\sigma}-\gamma
\end{aligned}
$$

They must either be both positive or both negative. However, factor (II) is nothing but $-\Lambda R_{r}$, which we want to be positive (for $\Lambda<0$ ). According to Eq. (25), the factor (II) is negative. It thus follows that we need factor (I) to also be negative. Since previously $\sigma<0$, the term $-2 \sigma$ in factor (I) is positive. The question is whether the factor (I) can be negative enough. The answer is no. In order to have the negative value for the factor (I), we need $\gamma\left(\sqrt{\gamma^{2}+\sigma}-\gamma\right)<2 \sigma$. Since $\gamma \geqslant \sqrt{-\sigma}$, we define $\gamma:=s \sqrt{-\sigma}$ in which $s \geqslant 1$. Replacing $\gamma=s \sqrt{-\sigma}$ in $\gamma\left(\sqrt{\gamma^{2}+\sigma}-\gamma\right)<2 \sigma$, we obtain

$$
s \sigma\left(s-\sqrt{s^{2}-1}\right)<2 \sigma .
$$

Since $\sigma<0$, this means we need

$$
s\left(s-\sqrt{s^{2}-1}\right)>2,
$$

which yields $s<-2 \sqrt{3} / 3$, contradicting $s \geqslant 1$. This shows that there is no black hole remnant for $\Lambda<0$ (or black hole remnant which is asymptotically de Sitter).

\section{BLACK HOLE REMNANT AND INFORMATION PARADOX IN ANTI-DE SITTER-LIKE UNIVERSE}

There are a few motivations for black hole remnants, one of which is that remnants prevent black holes from becoming arbitrarily hot during the end stage of the evaporation. Probably no one expects Hawking temperature to be truly divergent in the $M \rightarrow 0$ limit, but exactly what prevents just such a divergence is not agreed upon. One possibility is simply that new physics comes in at sufficiently high energy, thereby stopping black holes from evaporating further. Just such a possibility was investigated in [36] by appealing to the generalized uncertainty principle (GUP), which modifies quantum mechanics taking into account correction due to strong gravitational field. The remnant solution therein exhibits a rather peculiar property that its temperature is positive - how could a black hole be a remnant (not losing mass) yet continue to have Hawking radiation? One possible way out is to interpret this temperature as the internal energy of the remnant (since $E \sim k T$ ). The specific heat of the remnant is zero, and therefore it does not interact with the thermal environment [37, 38]. This means the remnant is stable, a pre-requisite for it to serve as dark matter candidate. Indeed, such a black hole remnant derived from GUP has been proposed as possible dark matter candidate [39].

Another virtue of black hole remnant is that it might be able to ameliorate the information paradox of black hole. The usual proposal to preserve quantum information is by having it scrambled and entangled in the Hawking radiation. Consider a black hole formed by a pure state. By unitarity one should recover pure state at the end of the black hole evaporation. The attempt to purify the Hawking radiation has given rise to issues like firewall [40]. The remnant picture, first proposed in [41], avoided this problem by proposing that Hawking radiation is never purified - states behind the horizon and states in the Hawking radiation remains mixed separately, but taken as a whole it is a pure state. Such a proposal is not without problems. For example, in order to hide plenty of quantum states behind the ever shrinking horizon, the BekensteinHawking entropy does not reflect all the interior degrees of freedom. There is also the infinite production problem. Both of these problems are discussed in details in [3]. The bottom line is that despite these issues, remnants should not be dismissed outright, and could well help to resolve the information paradox, especially if they have huge interiors due to non-trivial geometries. All these 
comments apply also to our massive gravity remnants, with the caveats that our remnants exist in anti-de Sitter spacetime.

There are two ways in which our remnants can be relevant to the information paradox. The first possibility is more straightforward: as we have explained in the introduction, our Universe could actually be asymptotically anti-de Sitter in the far future, with the current phase of acceleration caused by other fields [42] (This would also avoid the recently raised "swampland" issue of de Sitter space [43].). In [42], a quintessence was used. It is now appreciated that a simple quintessence model is difficult to be realized in string theory without fine tuning, so appealing to one to avoid the Swampland is swapping one difficulty with another [44-47]. However, other more complicated fields could still do the job [48].

Of course, there is the subtlety that the theory still needs to be coupled with these other fields and then strictly speaking the remnant solution would be different (if it still exists). If our Universe is asymptotically de Sitter, as most cosmologists believe, then our massive gravity remnants cannot be straightforwardly applied to understand actual black holes. Nevertheless, it is hoped that black hole remnants in the anti-de Sitter bulk may - eventually - help us to understand how information is preserved via holographic correspondence to a field theory on the conformal boundary.

The thermal stability of the massive gravity remnant is demonstrated by the fact that the heat capacity is zero, much like the remnant obtained from GUP mentioned above (this is not always the case for all GUP models, see e.g., [38]). This means that we have a thermodynamically inert and stable remnant. However, unlike the GUP remnant, its temperature is also zero. This is in fact much more natural - no mass is loss via Hawking emission and thus the remnant is stable. While we could argue that the GUP remnant temperature is really its internal energy, this feels somewhat contrived in comparison. Since our black hole has no electrical charge, the remnant is not like an extremal charged black hole, which could continue to radiate (despite having zero temperature) via nonthermal processes such as Schwinger pair production [3]; the remnant is arguably more stable and long-lived.

\section{DISCUSSIONS}

In this work we investigated whether dRGT massive gravity can admit remnant scenario, and found that it is indeed possible. To our knowledge, this is the first black hole remnant found in dRGT massive gravity. The black hole tends to zero temperature remnant with vanishing specific heat, at which point it stops evaporating and becomes stable. The remnant only has positive mass for $\gamma<0$. Massive gravity remnant could help to ameliorate the information paradox, modulo the usual challenges [3]. Here we discuss several issues and outlook for future works. Note that in [49], a solution in dyonic massive gravity was discussed in which there is a "remnant temperature", i.e. in the limit of vanishing radius, the temperature is nonzero - it is not a black hole remnant in the sense studied in this work.

In this work, we chose the reference metric

$$
f_{a b}=\operatorname{diag}\left(0,0, c^{2}, c^{2} \sin ^{2} \theta\right) .
$$

Since different reference metric might give different results, a more detailed analysis is required to find out how our results may change if another reference metric is chosen. In particular, although our result shows that remnants can only exist in asymptotically anti-de Sitter spacetime, other reference metric may allow remnants to exist in asymptotically de Sitter spacetime as well. This will require further investigations.

It is worth commenting on the cutoff scale of massive gravity theory. The cutoff scale of any theory is the scale beyond which the theory breaks down (in the sense of effective field theory), and one would expect new physics to come in at higher energy scale. The cutoff scale of general relativity (with massless graviton) is the Planck scale. As mentioned in [11], the cutoff scale for massive gravity is not the same as the strong-coupling scale of the theory. Furthermore, the latter need not necessarily mean that there is new physics, but only that perturbation theory breaks down. In fact strong gravity tends to raise the strong coupling scale [50]. Except from very near the singularities, black hole solutions we discussed here are valid in massive gravity theory.

Demonstrating that dRGT massive gravity admits black hole remnant solutions is only the first step in the analysis. One needs to consider the actual evolution of the black hole under Hawking evaporation. That is to say, one has to study the mass loss rate $\mathrm{d} M / \mathrm{d} t$. The importance of doing so is to check if the remnant state is attainable, i.e. if it can be reached in a finite time, such an analysis would be important to study the Page time [51-53] of the black hole. (Conversely, even if there is no remnant, one could have an "effective remnant" if the evaporation rate is infinite [38].) Presumably if the third law of black hole thermodynamics is valid for such black hole, it would take infinite amount of time to reach zero temperature state. In addition to the mass loss rate $\mathrm{d} M / \mathrm{d} t$, one should also study the sparsity of the Hawking radiation [38, 54, 55], which affects the lifetime of the black hole. This is beyond the scope of the current paper, and is left for future works.

As mentioned in Sec.(I), dRGT massive gravity suffers from a variety of problems, most notably the causality issue which plagues the theory with superluminal propagation and arbitrarily small closed causal curves, thus rendering the theory rather unpredictive. In addition, a "god-given" reference metric is somewhat unsatisfactory. These has led to the considerations of bimetric (HassanRosen) theory [15], in which the reference metric $f_{a b}$ is dynamical. Such a theory has some advantages over the original massive gravity [56], and its causal structures and constraints are gradually being understood [24, 57], though more research is clearly needed. 
Finally, let us speculate on the possibility that massive gravity remnants may be dark matter candidate. Black hole remnants as dark matter is of course not a new idea, see, e.g. [58-60] for some early examples. If our Universe is fundamentally anti-de Sitter, which the current phase of accelerated expansion caused by another field, say a quintessence, then it is possible that massive gravity remnants may play a role as dark matter. In addition, the idea that massive gravitons might be dark matter themselves had been proposed quite a few years back [61]. Massive gravitons remain possible as dark matter candidate in the context of bimetric gravity [62-65]. If remnants exist in that theory they could serve as an additional dark matter candidate.

\section{Acknowledgments}

We thank an anonymous referee for useful comments. BEP and SHH wish to thank the Shiraz University Re- search Council. The work of BEP has been supported financially by the Research Institute for Astronomy and Astrophysics of Maragha (RIAAM) under research project No. 1/5750-57. YCO thanks the National Natural Science Foundation of China (grant No.11705162) and the Natural Science Foundation of Jiangsu Province (No.BK20170479) for funding support. YCO also thanks Nordita, where this work was carried out, for hospitality during his summer visit for the Lambda Program. YCO also thank members of Center for Gravitation and Cosmology (CGC) of Yangzhou University (http://www.cgc-yzu.cn) for discussions.
[1] Steven B. Giddings, Phys. Rev. D 46 (1992) 1347, [arXiv:hep-th/9203059].

[2] Steven B. Giddings, "Black Holes and Massive Remnants", Phys. Rev. D 49 (1994) 4078, [arXiv:hep-th/9203059].

[3] Pisin Chen, Yen Chin Ong, Dong-han Yeom, "Black Hole Remnants and the Information Loss Paradox", Phys. Rep. 603 (2015) 1, [arXiv:1412.8366 [gr-qc]].

[4] Guido D'Amico, Claudia de Rham, Sergei Dubovsky, Gregory Gabadadze, David Pirtskhalava, Andrew J. Tolley, "Massive Cosmologies", Phys. Rev. D 84 (2011) 124046, [arXiv:1108.5231 [hep-th]].

[5] Benjamin P. Abbott et al. (LIGO Scientific Collaboration and Virgo Collaboration), "Observation of Gravitational Waves from a Binary Black Hole Merger", Phys. Rev. Lett. 116 (2016) 061102, [arXiv:1602.03837 [gr-qc]].

[6] Benjamin P. Abbott et al. (LIGO Scientific and Virgo Collaborations), "Tests of General Relativity with GW150914", Phys. Rev. Lett. 116 (2016) 221101, [arXiv:1602.03841 [gr-qc]].

[7] David G. Boulware, Stanley Deser, "Can Gravitation Have a Finite Range?", Phys. Rev. D 6 (1972) 3368.

[8] Markus Fierz, Wolfgang Pauli, "On Relativistic Wave Equations for Particles of Arbitrary Spin in an Electromagnetic Field", Proc. R. Soc. A 173 (1939) 211.

[9] Claudia de Rham, Gregory Gabadadze, "Generalization of the Fierz-Pauli Action", Phys. Rev. D 82 (2010) 044020, [arXiv:1007.0443 [hep-th]].

[10] Claudia de Rham, Gregory Gabadadze, Andrew J. Tolley, "Resummation of Massive Gravity", Phys. Rev. Lett. 106 (2011) 231101, [arXiv:1011.1232 [hep-th]].

[11] Claudia de Rham, "Massive Gravity", Living Rev. Relativ. 17 (2014) 7, [arXiv:1401.4173 [hep-th]].

[12] Sayed Fawad Hassan, Rachel A. Rosen, "On Non-Linear Actions for Massive Gravity", JHEP 07 (2011) 009, [arXiv:1103.6055 [hep-th]].

[13] Sayed Fawad Hassan, Rachel A. Rosen, "Resolving the Ghost Problem in Nonlinear Massive Gravity", Phys.
Rev. Lett. 108 (2012) 041101, [arXiv:1106.3344 [hep-th]].

[14] Sayed Fawad Hassan, Rachel A. Rosen, Angnis SchmidtMay, "Ghost-free Massive Gravity with a General Reference Metric", JHEP 02 (2012) 026, [arXiv:1109.3230 [hep-th]].

[15] S. F. Hassan, Rachel A. Rosen, "Bimetric Gravity from Ghost-free Massive Gravity", JHEP 02 (2012) 126 [arXiv:1109.3515 [hep-th]].

[16] David Vegh, "Holography Without Translational Symmetry", [arXiv:1301.0537].

[17] Mike Blake, David Tong, "Universal Resistivity from Holographic Massive Gravity", Phys. Rev. D 88 (2013) 106004, [arXiv:1308.4970 [hep-th]].

[18] Antonio De Felice, A. Emir Gumrukcuoglu, Chunshan Lin, Shinji Mukohyama, "On the Cosmology of Massive Gravity", Class. Quant. Grav. 30 (2013) 184004, [arXiv:1304.0484 [hep-th]].

[19] Stanley Deser, Keisuke Izumi, Yen Chin Ong, Andrew Waldron, "Massive Gravity Acausality Redux", Phys. Lett. B 726 (2013) 544, [arXiv:1306.5457 [hep-th]].

[20] Stanley Deser, McCullen Sandora, Andrew Waldron, George Zahariade, "Covariant Constraints for Generic Massive Gravity and Analysis of Its Characteristics", Phys. Rev. D 90 (2014) 104043, [arXiv:1408.0561 [hepth]].

[21] Stanley Deser, Keisuke Izumi, Yen Chin Ong, Andrew Waldron, "Problems of Massive Gravities", Mod. Phys. Lett. A30 (2015) 1540006, [arXiv:1410.2289 [hep-th]].

[22] Stanley Deser, Andrew Waldron, George Zahariade, "Propagation Peculiarities of Mean Field Massive Gravity", Phys. Lett. B 749 (2015) 144, [arXiv:1504.02919 [hep-th]].

[23] Pavel Motloch, Wayne Hu, Austin Joyce, Hayato Motohashi, "Self-Accelerating Massive Gravity: Superluminality, Cauchy Surfaces and Strong Coupling", Phys. Rev. D 92 (2015) 044024, [arXiv:1505.03518 [hep-th]].

[24] Sayed Fawad Hassan, Mikica Kocic, "On the Local Struc- 
ture of Spacetime in Ghost-Free Bimetric Theory and Massive Gravity", JHEP 05 (2018) 099, [arXiv:1706.07806 [hep-th]].

[25] Seyed Hossein Hendi, Gholam Hossein Bordbar, Behzad Eslam Panah, Shahram Panahiyan, "Neutron Stars Structure in the Context of Massive Gravity", JCAP 07 (2017) 004, [arXiv:1701.01039 [gr-qc]].

[26] Behzad Eslam Panah, Helei Liu, "White Dwarfs in de Rham-Gabadadze-Tolley Like Massive Gravity", Phys. Rev. D 99 (2019) 104074, [arXiv:1805.10650 [gr-qc]].

[27] Koushik Dutta, Ruchika, Anirban Roy, Anjan A. Sen, M.M. Sheikh-Jabbari, "Beyond $\Lambda$ CDM with Low and High Redshift Data: Implications for Dark Energy", [arXiv:1808.06623 [astro-ph.CO]].

[28] Kurt Hinterbichler, "Theoretical Aspects of Massive Gravity", Rev. Mod. Phys. 84 (2012) 671,[ arXiv:1105.3735 [hep-th]].

[29] Rong-Gen Cai, Ya-Peng Hu, Qi-Yuan Pan, Yun-Long Zhang, "Thermodynamics of Black Holes in Massive Gravity", Phys. Rev. D 91 (2015) 024032, [arXiv:1409.2369 [hep-th]].

[30] Sushant G. Ghosh, Lunchakorn Tannukij, Pitayuth Wongjun, "A Class of Black Holes in dRGT Massive Gravity and Their Thermodynamical Properties", Eur. Phys. J. C 76 (2016) 119, [arXiv:1506.07119 [gr-qc]].

[31] Seyed Hossein Hendi, Behzad Eslam Panah, Shahram Panahiyan, "Einstein-Born-Infeld-Massive Gravity: AdSBlack Hole Solutions and Their Thermodynamical Properties", JHEP 11 (2015) 157, [arXiv:1508.01311 [hep-th]].

[32] Ping Li, Xin-zhou Li, Ping Xi, "Black Hole Solutions in de Rham-Gabadadze-Tolley Massive Gravity", Phys. Rev. D 93 (2016) 064040, [arXiv:1603.06039 [gr-qc]].

[33] Hongsheng Zhang, Xin-Zhou Li, "Ghost Free Massive Gravity with Singular Reference Metrics", Phys. Rev. D 93 (2016) 124039, [arXiv:1510.03204 [gr-qc]].

[34] Yen Chin Ong, "Hawking evaporation time scale of topological black holes in anti-de Sitter spacetime", Nucl. Phys. B 903 (2016) 387, [arXiv:1507.07845 [gr-qc]].

[35] Yuan Yao, Meng-Shi Hou, Yen Chin Ong, "A complementary third law for black hole thermodynamics", Eur. Phys. J. C 79 (2019) 513, [arXiv:1812.03136 [gr-qc]].

[36] Ronald J. Adler, Pisin Chen, David I. Santiago, "The Generalized Uncertainty Principle and Black Hole Remnants", Gen. Rel. Grav. 33 (2001) 2101, [arXiv:gr-qc/0106080].

[37] Petr Jizba, Hagen Kleinert, Fabio Scardigli, "Uncertainty Relation on World Crystal and its Applications to Micro Black Holes", Phys. Rev. D 81 (2010) 084030, [arXiv:0912.2253 [hep-th]].

[38] Yen Chin Ong, "Zero Mass Remnant as an Asymptotic State of Hawking Evaporation", [arXiv:1806.03691 [grqc]].

[39] Pisin Chen, Ronald J. Adler, "Black Hole Remnants and Dark Matter", Nucl. Phys. Proc. Suppl. 124 (2003) 103, [arXiv:gr-qc/0205106].

[40] Ahmed Almheiri, Donald Marolf, Joseph Polchinski, James Sully, "Black Holes: Complementarity or Firewalls?", JHEP 02 (2013) 062, [arXiv:1207.3123 [hep-th]].

[41] Yakir Aharonov, Aharon Casher, Shmuel Nussinov, "The Unitarity Puzzle and Planck Mass Stable Particles", Phys. Lett. B 191 (1987) 51.

[42] Brett McInnes, "Quintessential Maldacena-Maoz Cosmologies", JHEP 04 (2004) 036, [arXiv:hep-th/0403104].

[43] Georges Obied, Hirosi Ooguri, Lev Spodyneiko, Cumrun Vafa, "De Sitter Space and the Swampland",
[arXiv:1806.08362 [hep-th]].

[44] Yashar Akrami, Renata Kallosh, Andrei Linde, Valeri Vardanyan, "The Landscape, the Swampland and the Era of Precision Cosmology", Fortsch. Phys. 67 (2019) 1800075, [arXiv:1808.09440 [hep-th]].

[45] Federico Tosone, Balakrishna S. Haridasu, Vladimir V. Luković, Nicola Vittorio, "Constraints on Field Flows of Quintessence Dark Energy", Phys. Rev. D 99 (2019) 043503, [arXiv:1811.05434 [astro-ph.CO]].

[46] Michele Cicoli, Senarath de Alwis, Anshuman Maharana, Francesco Muia, Fernando Quevedo, "De Sitter vs Quintessence in String Theory", Fortsch. Phys. 67 (2019) 1800079, [arXiv:1808.08967 [hep-th]].

[47] Mark P. Hertzberg, McCullen Sandora, Mark Trodden, "Quantum Fine-Tuning in Stringy Quintessence Models", Phys. Lett. B 797 (2019) 134878, [arXiv:1812.03184 [hepth]].

[48] Suddhasattwa Brahma, Md. Wali Hossain, "Dark Energy Beyond Quintessence: Constraints From the Swampland", JHEP 06 (2019) 070, [arXiv:1902.11014 [hep-th]].

[49] Seyed Hossein Hendi, Nematollah Riazi, Shahram Panahiyan, "Holographical Aspects of Dyonic Black Holes: Massive Gravity Generalization", Ann. Phys. (Berlin) 530 (2018) 1700211, [arXiv:1610.01505 [hep-th]].

[50] Jun Zhang, Shuang-Yong Zhou, "Can the Graviton Have a Large Mass Near Black Holes?", Phys. Rev. D 97 (2018) 081501, [arXiv:1709.07503 [gr-qc]].

[51] Don N. Page, "Average Entropy of a Subsystem", Phys. Rev. Lett. 71 (1993) 1291, [arXiv:gr-qc/9305007].

[52] Don N. Page, "Information in Black Hole Radiation", Phys. Rev. Lett. 71 (1993) 3743, [arXiv:hep-th/9306083].

[53] Don N. Page, "Time Dependence of Hawking Radiation Entropy", JCAP 09 (2013) 028, [arXiv:1301.4995 [hepth]].

[54] Finnian Gray, Sebastian Schuster, Alexander Van-Brunt, Matt Visser, "The Hawking Cascade from a Black Hole is Extremely Sparse", Class. Quant. Grav. 33 (2016) 115003, [arXiv:1506.03975 [gr-qc]].

[55] Matt Visser, Finnian Gray, Sebastian Schuster, Alexander Van-Brunt, "Sparsity of the Hawking Flux", Proceedings of the MG14 Meeting on General Relativity (2017); pp. 1724-1729, [arXiv:1512.05809 [gr-qc]].

[56] Yashar Akrami, S. F. Hassan, Frank Könnig, Angnis Schmidt-May, Adam R. Solomon, "Bimetric Gravity is Cosmologically Viable", Phys. Lett. B 748 (2015) 37, [arXiv:1503.07521 [gr-qc]].

[57] Sayed Fawad Hassan, Anders Lundkvist, "Analysis of Constraints and Their Algebra in Bimetric Theory", [arXiv:1802.07267 [hep-th]].

[58] Jane H. MacGibbon, "Can Planck-Mass Relics of Evaporating Black Holes Close the Universe?", Nature 329 (1987) 308.

[59] John D. Barrow, Edmund J. Copeland, Andrew R. Liddle, "The Cosmology of Black Hole Relics", Phys. Rev. D 46 (1992) 645.

[60] Bernard J. Carr, Jonathan H. Gilbert, James E. Lidsey, "Black Hole Relics and Inflation: Limits on Blue Perturbation Spectra", Phys. Rev. D 50 (1994) 4853, [arXiv:astro-ph/9405027].

[61] Sergei L. Dubovsky, Peter G. Tinyakov, Igor I. Tkachev, "Massive Graviton as a Testable Cold Dark Matter Candidate", Phys. Rev. Lett. 94 (2005) 181102, [arXiv:hepth/0411158].

[62] Katsuki Aoki, Shinji Mukohyama, "Massive Gravitons as 
Dark Matter and Gravitational Waves", Phys. Rev. D 94 (2016) 024001, [arXiv:1604.06704 [hep-th]].

[63] Eugeny Babichev, Luca Marzola, Martti Raidal, Angnis Schmidt-May, Federico Urban, Hardi Veermäe, Mikael von Strauss, "Gravitational Origin of Dark Matter", Phys. Rev. D 94 (2016) 084055, [arXiv:1604.08564 [hep-ph]].

[64] Eugeny Babichev, Luca Marzola, Martti Raidal, Angnis
Schmidt-May, Federico Urban, Hardi Veermäe, Mikael von Strauss, "Heavy Spin-2 Dark Matter", JCAP 09 (2016) 016, [arXiv:1607.03497 [hep-th]].

[65] Luca Marzola, Martti Raidal, Federico R. Urban, "Oscillating Spin-2 Dark Matter", Phys. Rev. D 97 (2018) 024010, [arXiv:1708.04253 [hep-ph]]. 